\title{
Short-Term Power Load Point Prediction Based on the Sharp Degree and Chaotic RBF Neural Network
}

\author{
Dongxiao Niu, Yan Lu, Xiaomin Xu, and Bingjie Li \\ School of Economics and Management, North China Electric Power University, Beijing 102206, China \\ Correspondence should be addressed to Yan Lu; hdluyan@163.com
}

Received 21 October 2014; Revised 9 December 2014; Accepted 16 December 2014

Academic Editor: Dan Simon

Copyright (C) 2015 Dongxiao Niu et al. This is an open access article distributed under the Creative Commons Attribution License, which permits unrestricted use, distribution, and reproduction in any medium, provided the original work is properly cited.

\begin{abstract}
In order to realize the predicting and positioning of short-term load inflection point, this paper made reference to related research in the field of computer image recognition. It got a load sharp degree sequence by the transformation of the original load sequence based on the algorithm of sharp degree. Then this paper designed a forecasting model based on the chaos theory and RBF neural network. It predicted the load sharp degree sequence based on the forecasting model to realize the positioning of short-term load inflection point. Finally, in the empirical example analysis, this paper predicted the daily load point of a region using the actual load data of the certain region to verify the effectiveness and applicability of this method. Prediction results showed that most of the test sample load points could be accurately predicted.
\end{abstract}

\section{Introduction}

Short-term load forecasting (STLF) plays a key role in power dispatching work. It is the foundation of power grid planning, grid control, system safety analysis, and the economic operation. The forecasting accuracy is closely related to the grid running safety and economy $[1,2]$. At present, many scholars and experts have already done a lot of theoretical researches and practical simulations on shortterm load forecasting [3-20]. More common short-term load forecasting models include regression prediction model [3], time series prediction model [4], artificial neural network prediction model [5], fuzzy logic and expert system [6, 7], the wavelet analysis model [8], chaos theory [9], and combination prediction model [10-12]. Literature [13] analyzed the power factors and predicted the short-term load based on rough set method. Literature $[14,15]$ introduced data mining techniques for short-term load forecasting. Literature [16] proposed a novel combination prediction model for short-term load forecasting which mainly studied the weight of combination prediction model with different scenarios. Literature [17] established a combination of modified firefly algorithm and support vector regression model which was used for the STLF. Literature [18] studied the short-term forecasting of categorical changes in wind power based on
Markov chain models. Literature [19] studied the short-term load forecasting based on the wavelet transform and grey model improved by PSO. Literature [20] proposed a relevance vector machine short-term load forecasting model based on nonnegative matrix decomposition.

On the whole, research on short-term load forecasting has been relatively mature. The models are more complicated and intelligent. Combination forecasting model is a research trend [21]. Inflection point is usually the most concern of the dispatch staff in short-term load forecasting. How to identify and predict inflection point of short-term load is a challenge problem. At present, there is no mature solution to the short-term load forecasting of inflection point. This paper gets a load sharp degree sequence by the transformation of the original load sequence based on the algorithm of sharp degree. Then, it forecasts the load sharp degree sequence by designing and training the forecasting model of chaotic RBF neural network to realize the short-term load forecasting of inflection point.

\section{Inflection Point Identification Algorithm Based on Sharp Degree}

Inflection point is the most important features of load curve. The inflection point in this paper is the point that 


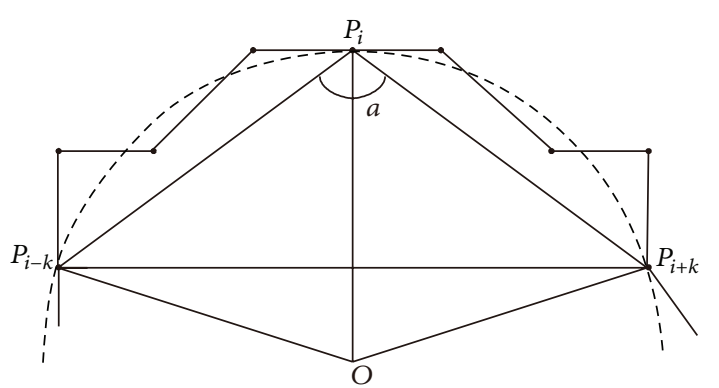

FIgURE 1: Part outline figure of the load curve.

changes the direction of load curve upward or downward in a certain time interval. This paper identifies the inflection point according to the sharp degree of each point on the load curve. Set the function of load curve as $P=p(t)$, in which the independent variable $t$ means time and the dependent variable $P$ represents the load time series.

Randomly select a point $P_{i}$ on the curve as the center. $P_{i-k}$ is the point in front of $P_{i}$ a distance of $k$. And $P_{i+k}$ is the point behind $P_{i}$ a distance of $k$.

Define the vector $k$ :

$$
\begin{gathered}
a_{i k}=(T(i)-T(i+k), P(i)-P(i+k)), \\
b_{i k}=(T(i)-T(i-k), P(i)-P(i-k)), \\
c_{i k}=(T(i-k)-T(i+k), P(i-k)-P(i+k)) .
\end{gathered}
$$

Define $\alpha$, the angle of $a_{i k}$ and $b_{i k}$, as the angle consisting of $P_{i-k}, P_{i}$, and $P_{i+k}$ on the load curve. Figure 1 shows the part outline figure of the load curve. The peripheral solid line is composed of load time series. The dotted line is the circular arc fitted by $P_{i-k}, P_{i}$, and $P_{i+k}$. And $O$ is the center of circular arc. Usually, the value of $k$ is set in the range of 35 . For the endpoint of load curve, we will take 3 to 5 sample values forward and backward on the sample load data. On the actual curve, the points $P_{i-k}, P_{i}$, and $P_{i+k}$ can be approximately regarded as three points on the circular arc as the interval of the three points is very small [22].

Assuming that $\left|P_{i} P_{i-k}\right|=\left|P_{i} P_{i+k}\right|$, then

$$
\begin{aligned}
\sin \left(\frac{\alpha}{2}\right) & =\frac{\left|P_{i-k} P_{i+k}\right| / 2}{\left|P_{i} P_{i-k}\right|}=\frac{\left|P_{i-k} P_{i+k}\right| / 2}{\left|P_{i} P_{i+k}\right|} \\
& =\frac{\left|P_{i-k} P_{i+k}\right|}{\left|P_{i} P_{i-k}\right|+\left|P_{i} P_{i+k}\right|} .
\end{aligned}
$$

In this function, $0<\alpha \leq 180$.

When $P_{i-k}, P_{i}$, and $P_{i+k}$ are in a straight line, $\alpha=180$, then

$$
\frac{\left|P_{i-k} P_{i+k}\right|}{\left|P_{i} P_{i-k}\right|+\left|P_{i} P_{i+k}\right|}=\sin \left(\frac{\alpha}{2}\right)=\sin (90)=1 \text {. }
$$

When the value of $\alpha$ decreases tending to zero,

$$
\frac{\left|P_{i-k} P_{i+k}\right|}{\left|P_{i} P_{i-k}\right|+\left|P_{i} P_{i+k}\right|}=\sin \left(\frac{\alpha}{2}\right)=\sin (0)=0 .
$$

Therefore, in a very small load curve, the variable sharp can be defined as sharp degree of $\alpha$

$$
\text { sharp }=1-\text { ang }=1-\frac{\left|P_{i-k} P_{i+k}\right|}{\left|P_{i} P_{i-k}\right|+\left|P_{i} P_{i+k}\right|} .
$$

The larger the value of sharp, the smaller the value of $\alpha$, and the curve would be more sharp. On the contrary, the curve would be more smooth. Because of the continuity of load, the identification of inflection point is closely related to the interval of load curve sequence and the set threshold value. Therefore, the threshold value can be set according to the load curve characteristics and actual situation. If sharp $\left(P_{i}\right)>T$, the $P_{i}$ can be regarded as inflection point.

\section{Prediction Model Based on Chaotic RBF Neural Network}

Artificial neural network is a common type of load forecasting methods. This method is highly intelligent which has strong ability to look for patterns [23]. Radical basis function (RBF) neural network is a kind of highly efficient feed-forward neural network. It has the best approximation performance and global optimal property. And the structure is simple; the training speed is fast [24]. Chaos prediction algorithm applied to power load is a very hot research field which is caused by the intrinsic characteristics of systems. It can be predicted in the short term [25]. It predicts directly according to the laws of the objective extracting data sequence itself and does not need to establish the mapping relation model of influence factors. In theory, it not only can decrease the cost of prediction, but can improve the prediction accuracy as well [26]. The method combining chaos and RBF neural network can make full use of the randomicity and initial value sensitivity of chaos. In addition, it can make full use of the function of RBF, such as massively parallel processing, self-organizing, and self-adapting [27].

Although sharp degree sequence is turbulent, it can be used to identify the inflection point. Chaotic time series are similar to random noise which is chaotic and unpredictable. But its inherent nonlinear dynamics structure makes it meet the short-term predictability [28]. Use phase space reconstruction to find the internal of regularity conversion sequences that can be described by a nonlinear mapping, and then approximate the nonlinear mapping by applying radial basis to forecast the inflection point. The process of the model established in this paper is shown in Figure 2.

First of all, we can get the sharp degree sequence which can identify the inflection point on the basis of Section 2 . Then, reconstruct phase space and identify chaos based on chaos theory. This paper selects mutual information method to calculate delay time, Cao method to determine embedding dimension, and the computing of largest Lyapunov exponent to identify the chaos. Thirdly, the input layer of RBF neural network can be determined based on the delay time and embedding dimension. Then, train the RBF neural network and predict the sharp degree sequence making use of the trained network. Finally, determine the threshold and position the inflection point. 


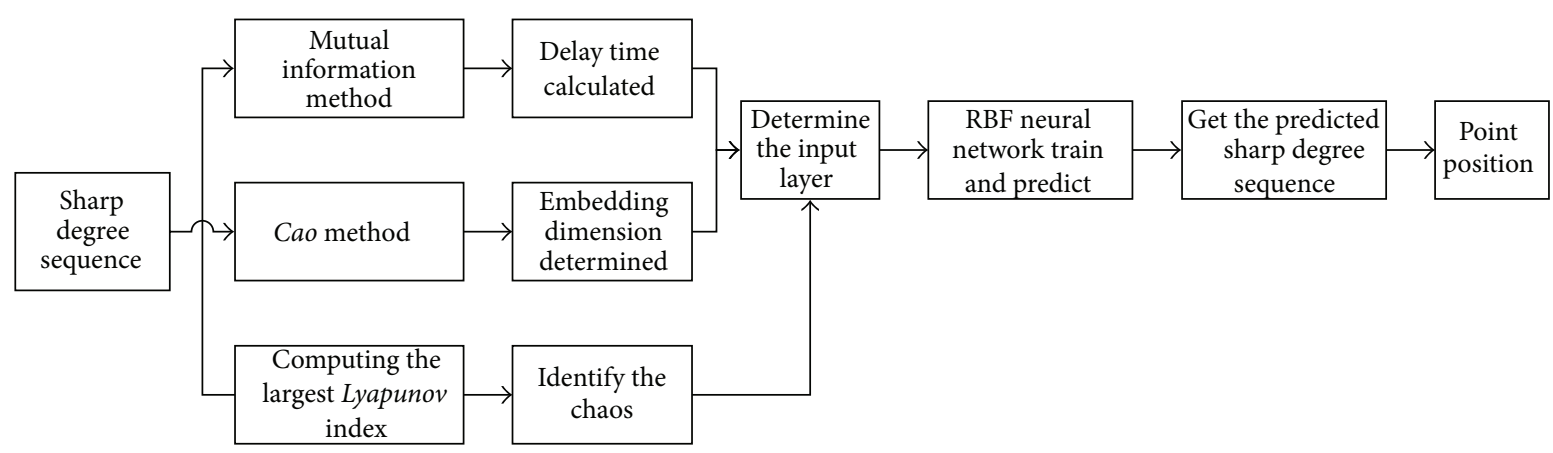

FIGURE 2: The steps of chaotic RBF neural network.

3.1. Phase Space Reconstruction and Chaos Identification. Extend the time series to three-dimension or higher dimension space by using delay-coordinate method to reconstruct state space which can find information and rules contained in the original time series. And by "Takens" theorem, the dynamic characteristic of attractor of the system can be restored in the sense of topological equivalence $[29,30]$. Assume that the time sequence is $x_{1}, x_{2}, x_{3}, \ldots, x_{n}$; properly select embedding dimension $m$ and time delay $\tau$; the reconstructed phase space is as follows:

$$
\begin{gathered}
Y_{1}=\left(x_{1}, x_{1+\tau}, \ldots, x_{1+(m-1) \tau}\right)^{T}, \\
Y_{2}=\left(x_{2}, x_{2+\tau}, \ldots, x_{2+(m-1) \tau}\right)^{T}, \\
\vdots \\
Y_{N}=\left(x_{N}, x_{N+\tau}, \ldots, x_{N+(m-1) \tau}\right)^{T},
\end{gathered}
$$

of which $N=n-(m-1) * \tau$ is the length of the vector sequence.

3.1.1. Delay Time Calculated by Mutual Information Method. Mutual information method is a kind of commonly used methods to calculate delay time [31]. It is suitable for large data sets and nonlinear problem. Set offline random variables $X, Y$. The mutual information $I(X, Y)$ is as follows:

$$
\begin{gathered}
I(X, Y)=H(X)+H(Y)-H(X, Y), \\
H(X)=\sum_{i=1}^{q} P\left(x_{i}\right) \log P\left(x_{i}\right) .
\end{gathered}
$$

In the functions, $P\left(x_{i}\right)$ is the probability of happening $x_{i}$, $q$ is the total number of possible things or states, and the logarithmic function often takes 2 for base number.

For the time series $x_{i}$ and delay time $\tau$ with an interval of $n$, set $P\left(x_{i}\right)$ as the probability of the $x_{i}$ in $\left\{x_{i}\right\}$, and $P\left(x_{i+\tau}\right)$, the probability of $x_{i+\tau}$ in $\left\{x_{i}\right\}$, can be calculated through the frequency in the corresponding time sequence, and $P\left(x_{i}, x_{i+\tau}\right)$, the joint probability of common presence of $x_{i}$ and $x_{i+\tau}$ in these two sequences, can be found in the corresponding grid on plane $\left(x_{i}, x_{i+\tau}\right)$. Therefore, the mutual information $I\left(x_{i}, x_{i+\tau}\right)$ can be regarded as the function of delay time $\tau, I(\tau)$. When the $I(\tau)$ meets the minimum for the first time, the lag time is the time delay of phase space reconstruction.

3.1.2. Embedding Dimension Determined by Cao Method. There are some methods to determine embedding dimension, such as the pseudo adjacent point method. G-P algorithm, and $C-C$ method. $C a o$ algorithm is a mature method which was put forward by Cao Liangyue [32]. When using Cao method, firstly set a delay time, and the embedding dimension and delay time are relatively independent [33]. Define

$$
a(i, m)=\frac{\left\|X_{i}(m+1)-X_{n(i, m)}(m+1)\right\|}{\left\|X_{i}(m)-X_{n(i, m)}(m)\right\|} .
$$

In the function, $X_{i}(m+1)$ is a vector that $i$ is in a space of $(m+$ 1) dimensions, $n(i, m)$ is the integer that makes $X_{n(i, m)}(m)$ be the nearest neighbor domain of $X_{i}(m)$ in the $m$-dimensional reconstruction space. $n(i, m)$ depends on $i$ and $m$; and $\|\bullet\|$ means the Euclidean distance.

Define the average of all $a(i, m)$ as

$$
E(m)=\frac{1}{N-m \tau} \sum_{i=1}^{N-m \tau} a(i, m) .
$$

Define the change of $m$ dimensional to $m+1$ dimensional as

$$
E 1(m)=\frac{E(m+1)}{E(m)} .
$$

If the time series are generated by the chaotic attractor, when $E 1(m)$ saturated with the increases of $m$, the value of $m+1$ is the minimum embedding dimension. Define $E 2(m)$ to distinguish deterministic chaotic signal and random signal:

$$
\begin{gathered}
E^{*}(m)=\frac{1}{N-m \tau} \sum_{i=1}^{N-m \tau}\left|x_{i+m \tau}-x_{n(i, m)+m \tau}\right|, \\
E 2(m)=\frac{E^{*}(m+1)}{E^{*}(m)} .
\end{gathered}
$$

For the random sequence, to all of the values of $m$, the values of $E 2(m)$ always equal about 1 , while for the chaotic sequence, the values of $E 2(m)$ are related to values of $m$. Namely, values of $E 2(m)$ are not constant to all of $m$ in chaotic sequence [34]. 
3.1.3. Identify the Chaos by Computing the Largest Lyapunov Exponent. The fundamental characteristics of chaos are the extreme sensitivity to initial values. The sensitivity can be measured by the exponent of Lyapunov. It reflects the divergence speed close to the initial orbit. The exponent of Lyapunov exponent reflects the contracting and tensile properties of the system in all directions. This paper calculates Lyapunov exponent based on the Wolf method to judge whether the original sequence is with chaos characteristics [35]. Wolf method is based on phase trajectory, phase plane, and phase volume. The process is as follows.

(1) Determine the embedding dimension and delay time to reconstruct phase space.

(2) Track the evolution of the distance from initial point and its closest point. Limit the short separation.

(3) Continue to the front steps until the end of the time series.

(4) Get the maximum Lyapunov exponent.

\subsection{Chaotic Time Series Predicted by RBF Neural Network}

3.2.1. The Basic Principle of RBF Neural Network. RBF neural network is a feed-forward neural network consisting of input layer, hidden layer, and output layer $[36,37]$, as shown in Figure 3.

Input layer to hidden layer is linear connection. And hidden layer to output layer is nonlinear connection.

3.2.2. Chaotic RBF Neural Network Prediction Steps. The specific steps of chaotic RBF neural network prediction are as follows.

(1) Establish the Network. Determine the input and output according to the calculated embedding dimension $m$ and delay time $\tau$. The input is a vector $\left[x_{i}, x_{i+\tau}, \ldots, x_{i+(m-1) \tau}\right]$. The output is a data $x_{2+(m-1) \tau}$. RBF neural network is a mapping from $m$-dimension to 1 -dimension. In this paper, the number of input layer nodes is $m$. The number of output layer nodes is 1. The input matrix $X$ and output matrix $Y$ are as follows:

$$
\begin{gathered}
X=\left[\begin{array}{cccc}
x_{1} & x_{1+\tau} & \cdots & x_{1+(m-1) \tau} \\
x_{2} & x_{2+\tau} & \cdots & x_{2+(m-1) \tau} \\
\cdots & \cdots & \cdots & \vdots \\
x_{n-(m-1) \tau} & x_{n-(m-2) \tau} & \cdots & x_{n}
\end{array}\right], \\
Y=\left[\begin{array}{c}
x_{2+(m-1) \tau} \\
x_{3+(m-1) \tau} \\
\vdots \\
x_{n+1}
\end{array}\right] .
\end{gathered}
$$

(2) Training Stage. Select a certain length of time series; set matrix $X$ as input and vector $Y$ as output to train the network.

(3) Forecasting Stage. Set $X^{\prime}=\left[x_{n+1-(m-1) \tau}, x_{n+1-(m-2) \tau}, \ldots\right.$, $\left.x_{n+1}\right]$ as the input of the neural network, and the output

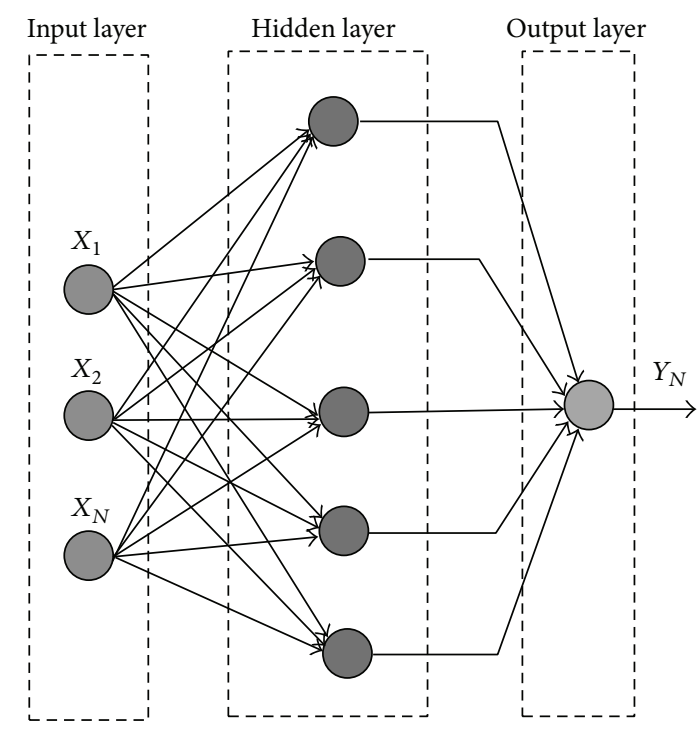

FIGURE 3: RBF neural network structure.

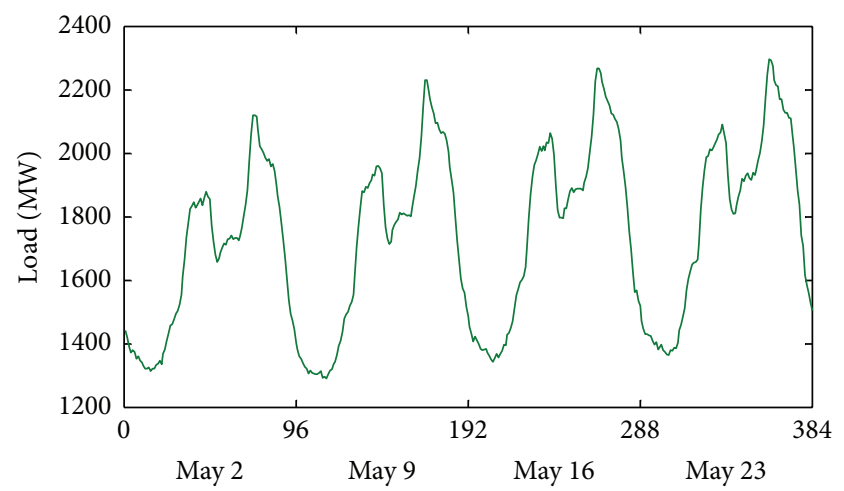

FIgURE 4: The original load trend chart of a region.

$Y^{\prime}=x_{n+2}$ is the actual predictive value of the sequence. Then iterate the network with the predictive value as a new network input.

\section{The Empirical Example Analysis}

To do empirical analysis, this paper selects the load on May 2, 2011, May 9, May 16, and May 23, and May 30 (Monday) of a region and collects 96 points per day, a total of 480 load data to calculate. Among them, set the data of first four days, a total of 384 data as the training sample, the data of last day, and a total of 96 as test samples, to verify and analyze the load prediction model of inflection points.

Firstly, compose the load data of May 2, 9, 16, and 23 to a load sequence. Then, calculate the sharp degree of every point of the load data according to the identification algorithm of inflection point based on sharp degree that was mentioned above. The load sequence and sharp degree sequence are, respectively, shown in Figures 4 and 5.

As can be seen from Figure 5, the polarization phenomenon of sharp degree sequence is obvious. The point 


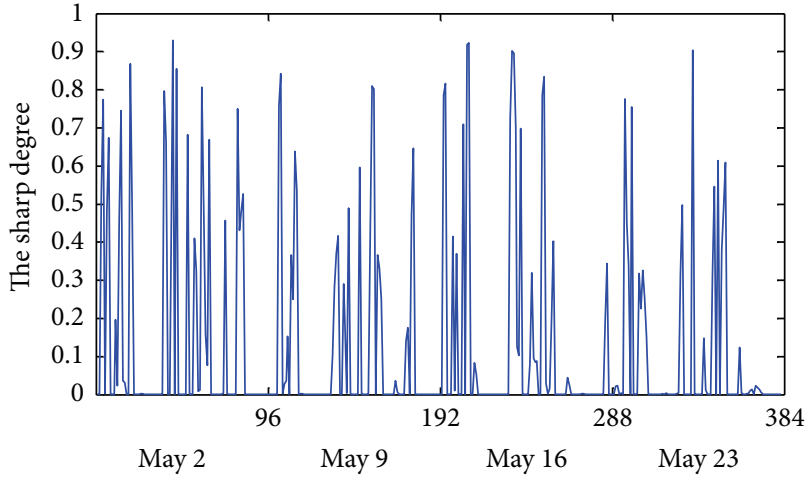

Figure 5: The sharp degree of load sequence diagram chart.

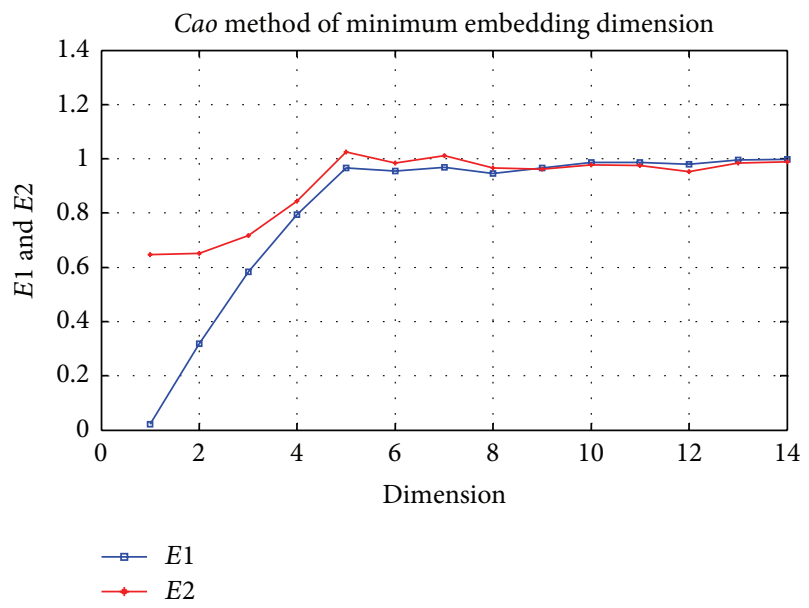

FIGURE 6: Cao method for minimum embedding dimension.

of sharp degree sequence is corresponding to the point of original sequence. Most of the values in Figure 5 are less than 0.1 , which are corresponding to the flat load curve in Figure 4. Only a few values in Figure 5 are between 0.1 and 1, which are corresponding to the inflection points in Figure 4. Therefore set the threshold as 0.1 . The load point with a sharp degree value greater than 0.1 is the inflection point defined in this paper. Thus, inflection point can be effectively identified by the sharp degree sequence.

According to the mutual information method, the delay time $\tau=4$. And calculate the minimum embedding dimension with Cao method. It can be seen in Figure 6 that $E 1$ tends to be saturate along with the increase of $m$. When $m \geq 5, E 1$ remains the same. So the minimum embedding dimension is $6(m=5+1=6)$. At the same time, it also can be seen in the diagram that, with different values of $m$, E2 is not always equal to 1. The value of Lyapunov exponent is 0.3017 after being calculated by Wolf method, which is greater than zero. So the conversion sequences of input can be judged as chaotic sequence.

Reconstruct the phase space according to the minimum embedding dimension and delay time. Then we can predict the sharp degree of load sequence based on RBF neural network. The most critical part of determining RBF network

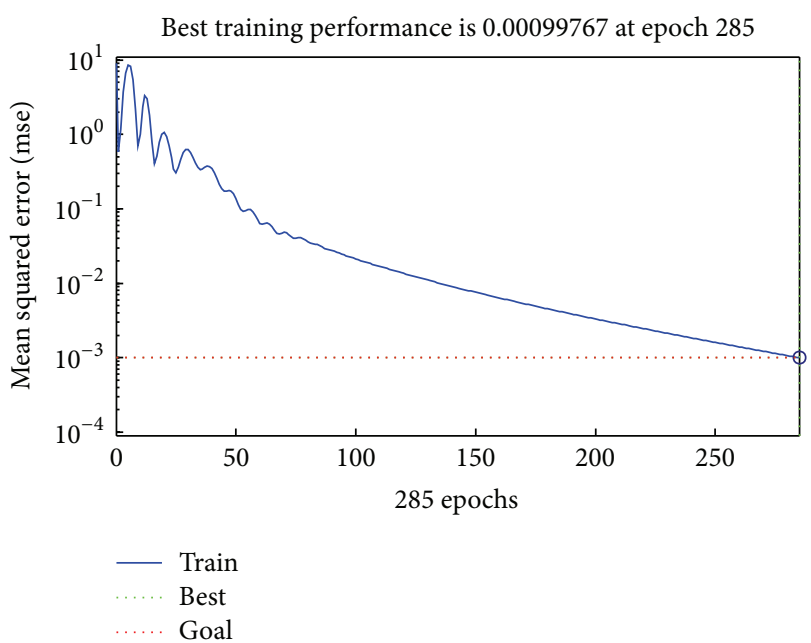

FIGURE 7: The convergence curve in training process.

is the choice of nonlinear excitation function. In some typical excitation functions, this paper uses the Gaussian function as the excitation function for its radial symmetry, smoothness, and simple expression formula. Set the error of training system to 0.001 . Select the self-organizing selection center method to determine the network center, width, and weight. Then train the training sample with RBF neural network. The convergence curve in training process is shown in Figure 7. We can see that the blue trace is the convergence curve of training. The red horizontal line which parallels the $x$-axis and equals $10^{-3}$ is the goal of training. The green vertical line which parallels the $y$-axis and equals 285 epochs at the far right side of the plot means that best training performance is 0.00099767 at epoch 285 . The forecasting result of test samples of the load sharp degree sequence is shown in Figure 8.

This paper uses the average absolute percentage error MAPE and the root mean square error RMSE to evaluate the forecasting effect, and the formula is

$$
\begin{gathered}
\text { MAPE }=\frac{1}{N} \sum_{n=1}^{N n}\left|\frac{\hat{y}_{n}-y_{n}}{y_{n}}\right|, \\
\operatorname{RMSE}=\left(\frac{1}{N} \sum_{n=1}^{N}\left(\hat{y}_{n}-y_{n}\right)^{2}\right)^{1 / 2} .
\end{gathered}
$$

The calculation shows that the MAPE is $5.293 \%$ and the RMSE is $6.391 \%$. The predicting is with a high accuracy.

The forecasting error curve of load sharp degree sequence test samples based on chaotic RBF is shown in Figure 9. It can be seen that there is one point whose error value is bigger than others. The point is the ninth point of prediction sequence. The actual value of this point is less than 0.001 . However, the predicted value is 0.576 . The reason of this situation is that the ninth point of 96 points in the historical everyday data is inflection point. The ninth point of forecasting sequence is an abnormal point. 


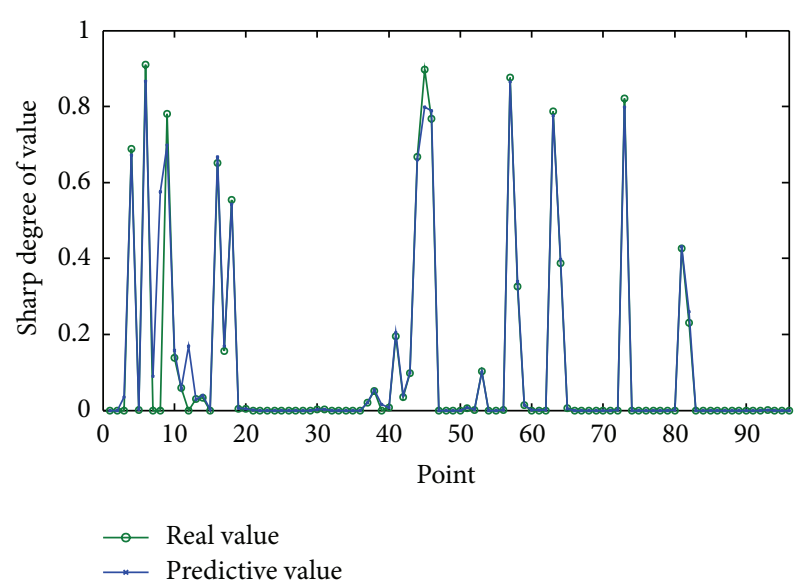

FIGURE 8: Contrast figure of chaotic RBF inflection point sequence predicted values and the real values.

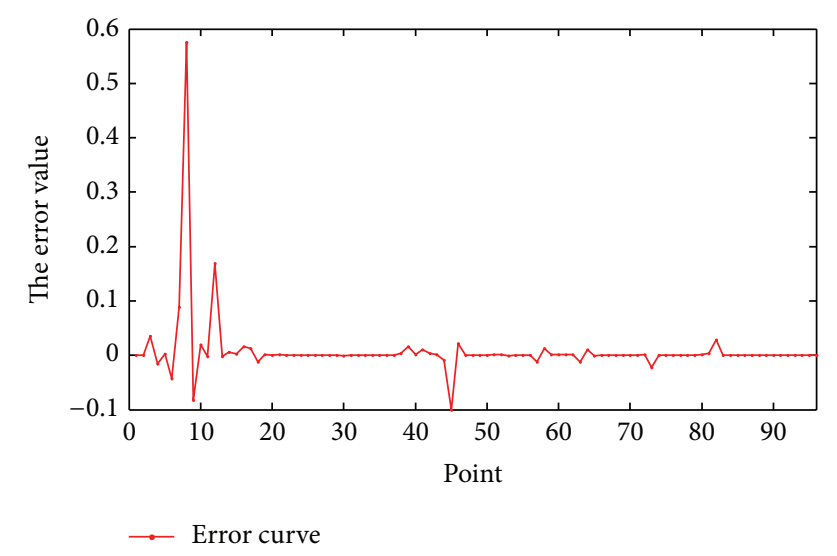

FIGURE 9: Error graph of chaotic RBF inflection.

This paper selects BP neural network model in order to verify the effectiveness and superiority of the proposed algorithm. BP neural network is one of the most commonly used kinds of artificial neural network. The forecasting result of test samples based on chaotic RBF and BP is shown in Figure 10. The MAPE of the results based on BP neural network is $9.149 \%$, which is much higher than the MAPE of chaotic RBF. Thus, the superiority of chaotic RBF algorithm is evident particularly.

Set the inflection point threshold $T=0.1$, extract the points with a predictive value greater than 0.1 in sharp degree sequence, and then locate and highlight the points in the known load curve on May 30, as shown in Figure 11.

It can be seen from Figure 11 that most of the inflection points of test samples can be accurately predicted by either using inflection point identification algorithm based on contour sharp degree or forecasting model based on chaotic RBF neural network and that verified that inflection point prediction model proposed in this paper is of high accuracy.

In order to further validate the applicability and effectiveness of model, this paper selected representative load curves of different seasons to carry out the empirical researches.

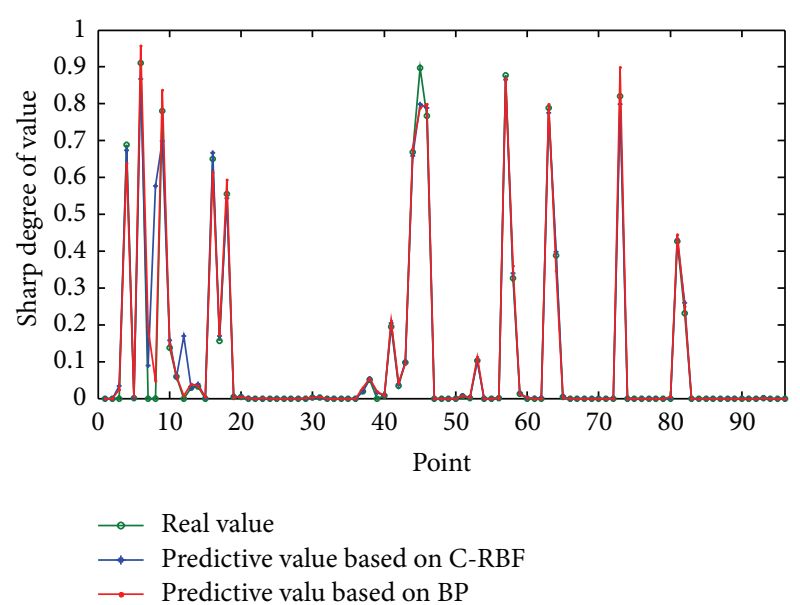

FIGURE 10: Contrast figure of the real values, predicted values based on chaotic RBF, and predicted values based on BP.

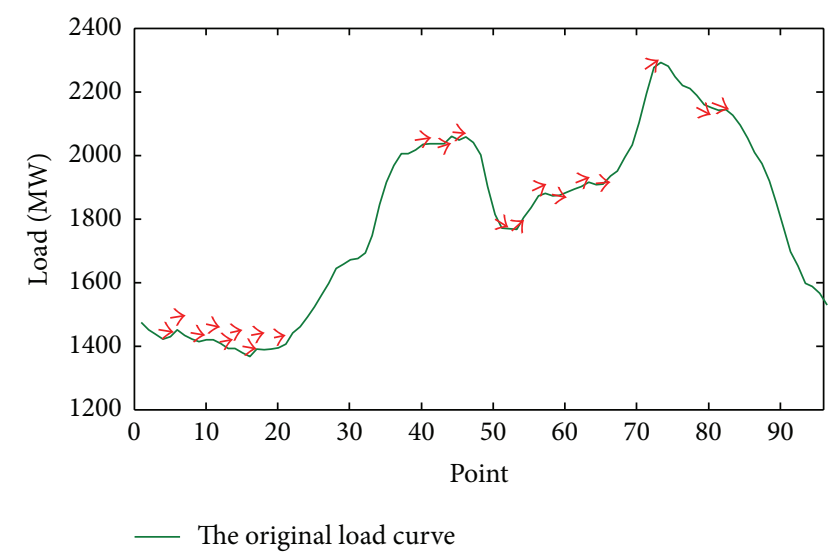

FIGURE 11: The inflection point location map according to the predicted results.

The prediction training sample contains 96 points per day of the same week type 4 weeks before the prediction, and the training data totally contains 384 points. The prediction error results are shown in Table 1.

The forecasting of 16 groups data shows that the average MAPE of the model proposed in this paper is $4.25 \%$. The average RMSE is $3.897 \%$. It suggests that the forecast effect is fine and the model is with strong practicability and applicability.

\section{Conclusion}

This paper puts forward a feasible load inflection point prediction method by proposing an inflection point forecasting model that can realize effective prediction of inflection point based on the algorithm of sharp degree and chaotic RBF neural network on the basis of research on short-term power load.

In the calculation example, the paper proposed an empirical analysis method of 96 points per day, and the algorithm can be also applied to the inflection point prediction of a 
TABLE 1: Results of empirical study in 2011.

\begin{tabular}{lccccccc}
\hline Number & Time & MAPE & RMSE & Number & Time & MAPE & RMSE \\
\hline 1 & January 3 & 6.322 & 2.647 & 9 & August 9 & 3.095 \\
2 & January 11 & 3.769 & 5.97 & 10 & August 16 & 3.616 \\
3 & January 19 & 6.937 & 3.969 & 11 & August 25 & 2.31 \\
4 & January 28 & 4.723 & 6.162 & 12 & August 31 & 3.202 & 5.967 \\
5 & May 4 & 3.11 & 2.217 & 13 & October 8 & 2.459 \\
6 & May 18 & 8.468 & 4.392 & 14 & October 19 & 4.193 \\
7 & May 24 & 4.271 & 2.344 & 15 & October 22 & 2.719 \\
8 & May 31 & 5.187 & 6.153 & 16 & October 28 & 3.967 \\
\hline \multicolumn{5}{c}{ The mean of RMSE } \\
\hline
\end{tabular}

shorter or a longer time interval. However, the time interval and different threshold selection will directly affect the inflection point identification based on the forecasted sharp degree sequence. According to actual needs, the original load time interval and the value of threshold can be adjusted.

Although forecasting of the load curve contains the information of inflection point, during the ordinary load forecasting, the prediction error of inflection points is slightly larger than other points. This paper transformed the original load sequence based on the sharp degree. And after transformation, the polarization phenomenon of the sequence is obvious that highlights the regularity of the change of the inflection point. In the later research work, the inflection point forecast correction, the improvement of original load curve forecasting results, and mining of the influence factors of inflection point to increase the input dimension of inflection point prediction can be considered to further improve the prediction accuracy.

\section{Conflict of Interests}

The authors declare that there is no conflict of interests regarding the publication of this paper.

\section{Acknowledgment}

This paper is supported by the National Science Foundation of China (Grant nos. 71071052 and 71471059).

\section{References}

[1] D. X. Niu, S. H. Cao, and J. C. H. Lu, Power Load Forecasting Technique and Its Application, China Electric Power Press, 2009.

[2] C. H. Q. Kang, Q. Xia, and M. Liu, Power System Load Forecasting, China Electric Power Press, 2007.

[3] T. Hong and P. Wang, "Fuzzy interaction regression for short term load forecasting," Fuzzy Optimization and Decision Making, vol. 13, no. 1, pp. 91-103, 2014.

[4] N. Amjady, "Short-term hourly load forecasting using timeseries modeling with peak load estimation capability," IEEE Transactions on Power Systems, vol. 16, no. 3, pp. 498-505, 2001.

[5] L. Hernández, C. Baladrón, J. M. Aguiar et al., "Artificial neural networks for short-term load forecasting in microgrids environment," Energy, vol. 75, pp. 252-264, 2014.
[6] M. Y. Chow and H. Tram, "Application of fuzzy logic technology for spatial load forecasting," IEEE Transactions on Power Systems, vol. 12, no. 3, pp. 1360-1366, 1997.

[7] D. Srinivasan and S. S. Tan, "Parallel neural network-fuzzy expert system strategy for short-term load forecasting: system implementation and performance evaluation," IEEE Transactions on Power Systems, vol. 14, no. 3, pp. 1100-1106, 1999.

[8] W. J. Staszewski and K. Worden, "Wavelet analysis of timeseries: coherent structures, chaos and noise," International Journal of Bifurcation and Chaos, vol. 9, no. 3, pp. 455-471, 1999.

[9] H. Mori and S. Vrano, "Short-term load forecasting with chaos time series analysis," in Proceedings of the International Conference on Intelligent Systems Applications to Power Systems, pp. 283-287, 2007.

[10] J. X. Che and J. Z. Wang, "Short-term load forecasting using a kernel-based support vector regression combination model," Applied Energy, vol. 132, pp. 602-609, 2014.

[11] A. Deihimi, O. Orang, and H. Showkati, "Short-term electric load and temperature forecasting using wavelet echo state networks with neural reconstruction," Energy, vol. 57, pp. 382401, 2013.

[12] H. J. Sadaei, R. Enayatifar, A. H. Abdullah, and A. Gani, "Shortterm load forecasting using a hybrid model with a refined exponentially weighted fuzzy time series and an improved harmony search," International Journal of Electrical Power and Energy Systems, vol. 62, pp. 118-129, 2014.

[13] Z. H. Xiao and S. J. Ye, "Rough set method for short-term load forecasting," Journal of Systems Engineering, vol. 24, no. 2, pp. 143-149, 2009.

[14] C. H. Kim, B. G. Koo, and J. H. Park, "Short-term electric load forecasting using data mining technique," Journal of Electrical Engineering and Technology, vol. 7, no. 6, pp. 807-813, 2012.

[15] L. Z. H. Zhu, "Short-term electric load forecasting with combined data mining algorithm," Automation of Electric Power Systems, vol. 30, no. 14, pp. 82-86, 2006.

[16] D. X. Niu and Y. N. Wei, "Short-term power load combinatorial forecast adaptively weighted by FHNN similar-day clustering," Automation of Electric Power Systems, vol. 37, no. 3, pp. 54-57, 2013.

[17] A. Kavousi-Fard, H. Samet, and F. Marzbani, "A new hybrid Modified Firefly Algorithm and Support Vector Regression model for accurate Short Term Load Forecasting," Expert Systems with Applications, vol. 41, no. 13, pp. 6047-6056, 2014.

[18] M. Yoder, A. S. Hering, W. C. Navidi, and K. Larson, "Shortterm forecasting of categorical changes in wind power with Markov chain models," Wind Energy, vol. 17, no. 9, pp. 14251439, 2014. 
[19] S. Bahrami, R.-A. Hooshmand, and M. Parastegari, "Short term electric load forecasting by wavelet transform and grey model improved by PSO (particle swarm optimization) algorithm," Energy, vol. 72, pp. 434-442, 2014.

[20] S. H. D. Pan, Z. H. N. Wei, Z. H. Gao et al., "A short-term load forecasting model based on relevance vector machine with nonnegative matrix factorization," Automation of Electric Power Systems, vol. 36, no. 11, pp. 62-66, 2012.

[21] Q.-H. Zhang, "A model for short-term load forecasting in power system based on multi-AI methods," System Engineering Theory and Practice, vol. 33, no. 2, pp. 354-362, 2013.

[22] Z. G. Qian and X. Z. Lin, "Detection algorithm of image corner based on contour sharp degree," Computer Engineering, vol. 34, no. 6, pp. 202-204, 2008.

[23] B. Amrouche and X. Le Pivert, "Artificial neural network based daily local forecasting for global solar radiation," Applied Energy, vol. 130, pp. 333-341, 2014.

[24] R. Mohammadi, S. M. T. F. Ghomi, and F. Zeinali, "A new hybrid evolutionary based RBF networks method for forecasting time series: a case study of forecasting emergency supply demand time series," Engineering Applications of Artificial Intelligence, vol. 36, pp. 204-214, 2014.

[25] H. Y. Luo, T. Q. Liu, and X. Y. Li, "Chaotic forecasting method of short-term wind speed in wind farm," Power System Technology, vol. 33, no. 9, pp. 67-71, 2009.

[26] M. D. Alfaro, J. M. Sepúlveda, and J. A. Ulloa, "Forecasting chaotic series in manufacturing systems by vector support machine regression and neural networks," International Journal of Computers Communications \& Control, vol. 8, no. 1, pp. 8-17, 2013.

[27] Z. Y. Zhang, T. Wang, and X. G. Liu, "Melt index prediction by aggregated RBF neural networks trained with chaotic theory," Neurocomputing, vol. 131, pp. 368-376, 2014.

[28] H. B. Bi and Y. B. Zhang, "Application of the chaotic RBF neural network on electrical loads prediction," Science, Technology and Engineering, vol. 9, no. 24, pp. 7480-7492, 2009.

[29] W. Y. Zhang, W. C. Hong, Y. Dong, G. Tsai, J. T. Sung, and G. F. Fan, "Application of SVR with chaotic GASA algorithm in cyclic electric load forecasting," Energy, vol. 45, no. 1, pp. 850858, 2012.

[30] S. Kouhi, F. Keynia, and S. N. Ravadanegh, "A new short-term load forecast method based on neuro-evolutionary algorithm and chaotic feature selection," International Journal of Electrical Power \& Energy Systems, vol. 62, pp. 862-867, 2014.

[31] X.-Q. Lu, B. Cao, M. Zeng, S.-S. Huang, and X.-G. Liu, "Algorithm of selecting delay time in the mutual information method," Chinese Journal of Computational Physics, vol. 23, no. 2, pp. 184-188, 2006.

[32] K. Aihara, T. Takabe, and M. Toyoda, "Chaotic neural networks," Physics Letters A, vol. 144, no. 6-7, pp. 333-340, 1990.

[33] Y. R. Cheng and S. B. Guo, "Stock price prediction based on analysis of chaotic time series," Journal of UEST of China, vol. 32, no. 4, pp. 469-472, 2003.

[34] S.-Q. Zhang, J. Jia, M. Gao, and X. Han, "Study on the parameters determination for reconstructing phase-space in chaos time series," Acta Physica Sinica, vol. 59, no. 3, pp. 1576$1582,2010$.

[35] D. Kressner, M. Pleinger, and C. Tobler, "A preconditioned lowrank CG method for parameter-dependent Lyapunov matrix equations," Numerical Linear Algebra with Applications, vol. 21, no. 5, pp. 666-684, 2014.
[36] J. Z. Zhou, Y. C. Zhang, Q. Q. Li, and J. Guo, "Probabilistic shortterm load forecasting based on dynamic self-adaptive radial basis function network," Power System Technology, vol. 34, no. 3, pp. 37-41, 2010.

[37] H. L. Deng and X. Q. Li, "Stock price inflection point prediction method Based on chaotic time series analysis," Statistics and Decision, no. 5, pp. 19-20, 2007. 


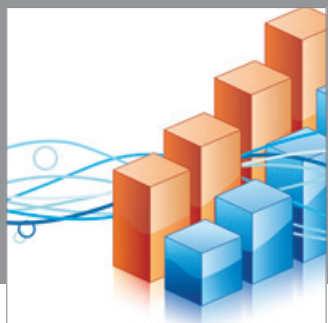

Advances in

Operations Research

mansans

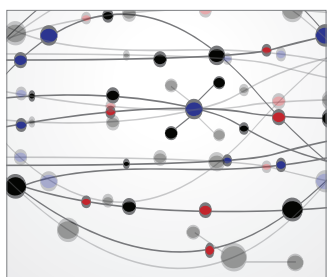

The Scientific World Journal
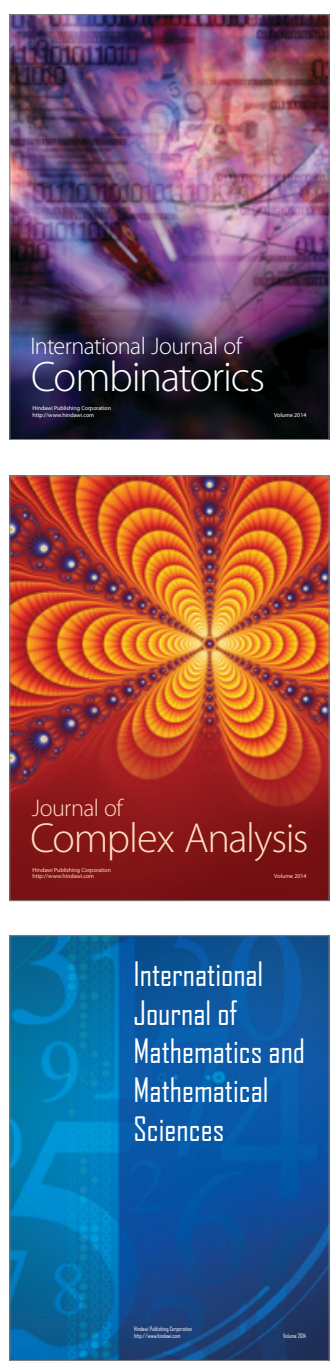
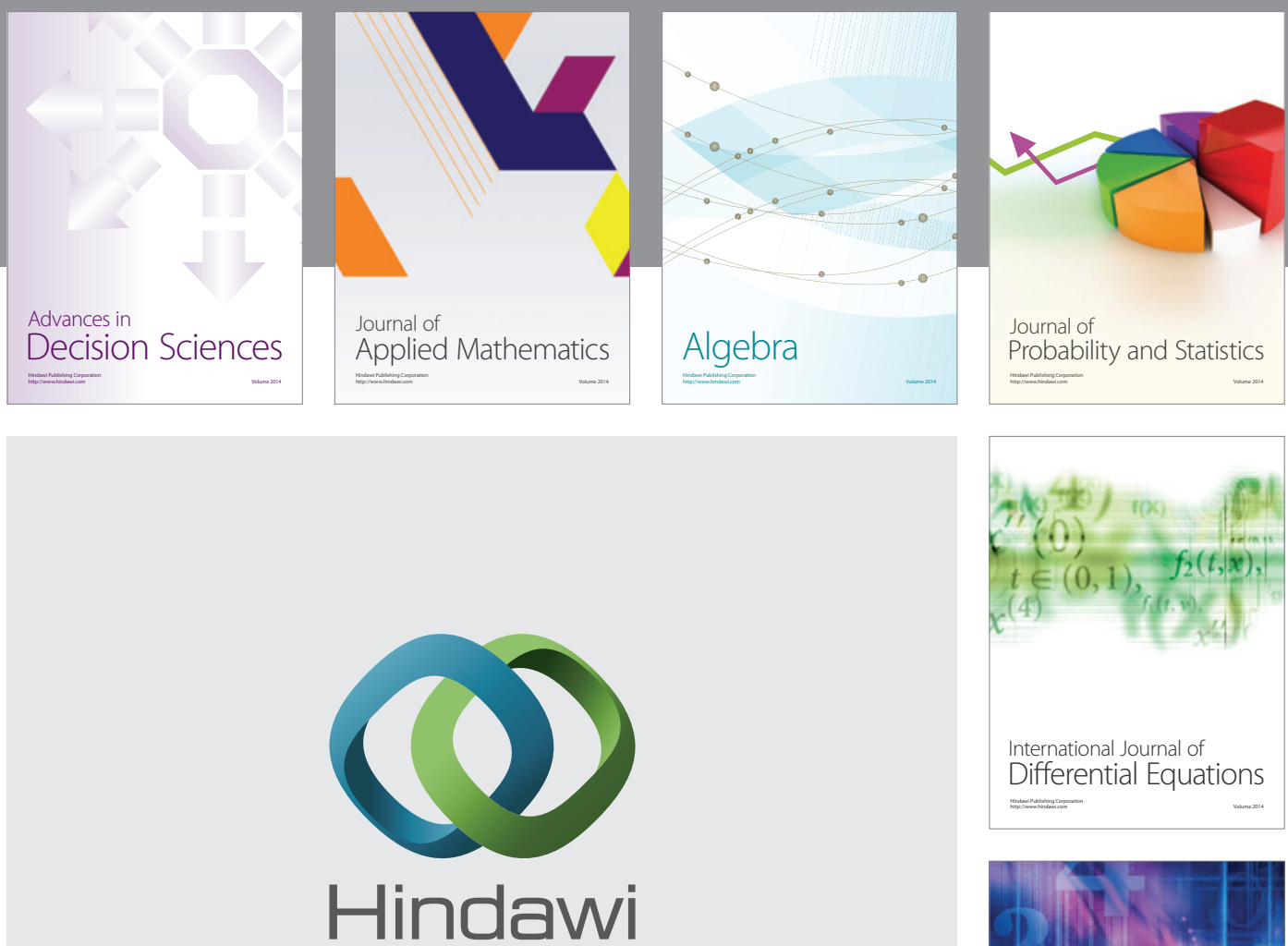

Submit your manuscripts at http://www.hindawi.com
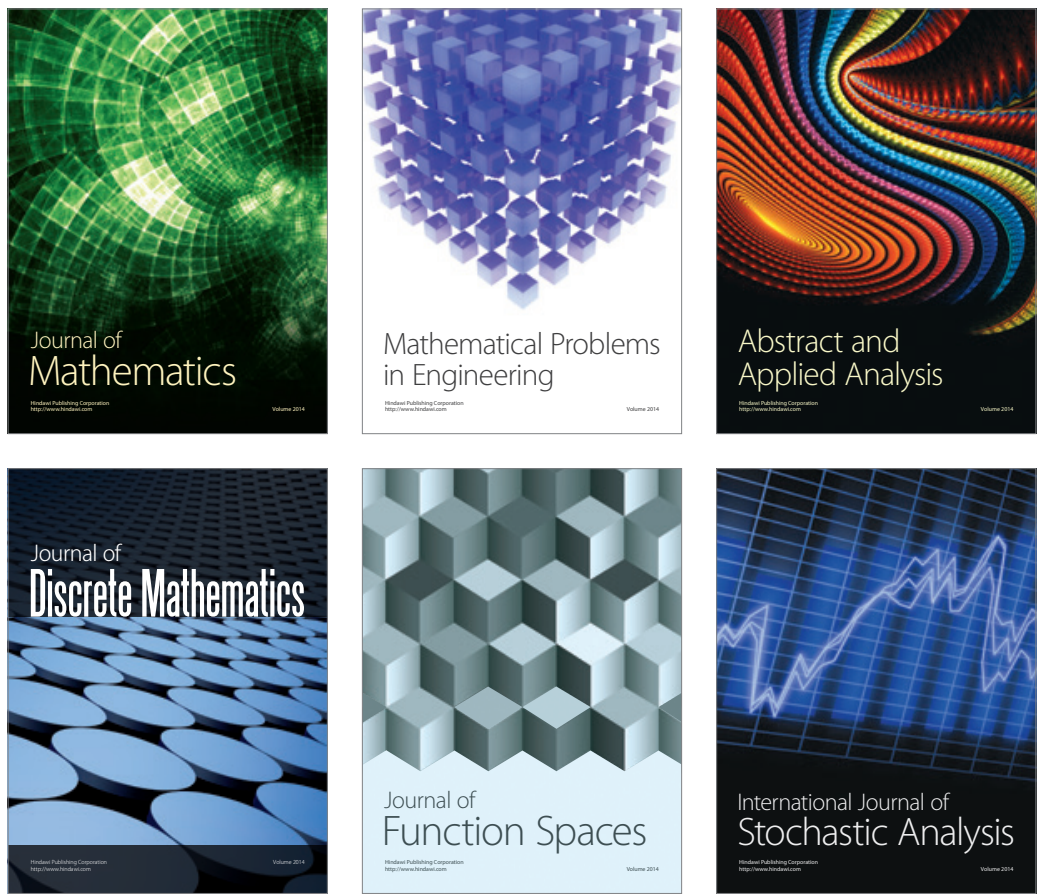

Journal of

Function Spaces

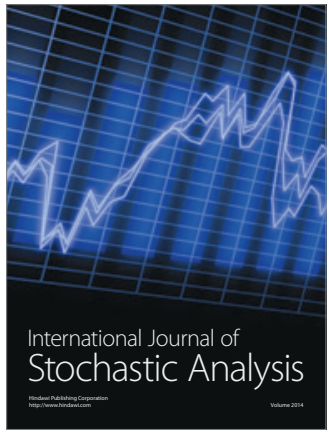

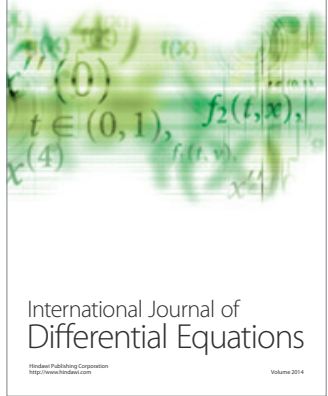
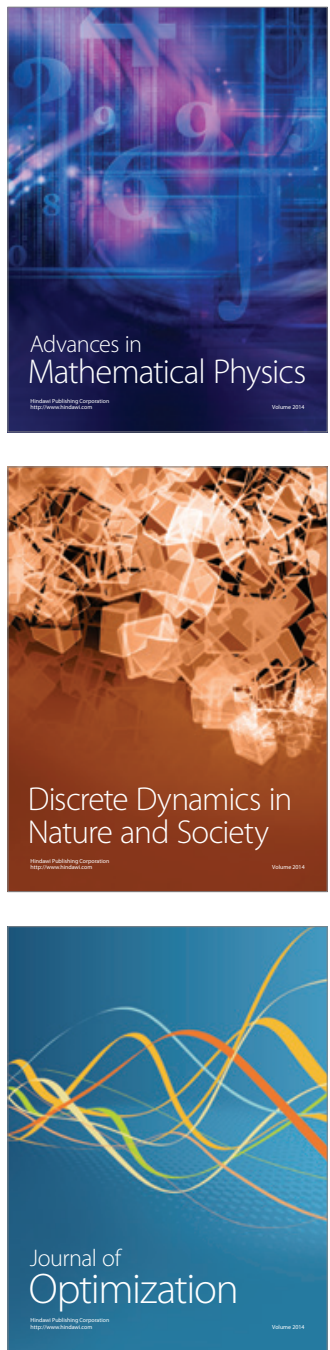